\title{
VARIAÇÃO GEOGRÁFICA DE CARACTERES QUANTITATIVOS EM OGCOCEPHALUS VESPERTILIO (LINNAEUS) (TELEOSTEI, LOPHIIFORMES, OGCOCEPHALIDAE)
}

\author{
Mauro José Cavalcanti ${ }^{1}$ \\ Paulo Roberto Duarte Lopes ${ }^{2}$
}

\begin{abstract}
GEOGRAPHIC VARIATION OF MORPHOMETRIC CHARACTERS IN OGCOCEPHALUS VESPERTILIO (LinNAeus) (Teleostei, Lophifformes, Ogcocephalidae). Patterns of geographic variation in 10 morphometric characters were analyzed in a sample of 91 specimens of the batfish, Ogcocephalus vespertilio (L.), from the NE and SE Brazilian coast, using multivariate statistics and randomization tests. The specimens were ordinated by principal components analysis in two groups corresponding to the regions north and south of the $23^{\circ} \mathrm{C}$ isotherm, and size variation was found to account for $70.3 \%$, whereas shape differences accounted for $23.6 \%$ of the total variation in morphometric characters. The two groups were different at the $1 \%$ significance level by multivariate analysis of variance based on the Wilk's criterion, tested by a randomization procedure. Width of illicial cavity and distance from anus to anal fin were the characters most contributing to the differentiation of the population samples. KEY WORDS. Ogcocephalidae, Ogcocephalus, geographic variation, multivariate morphometrics, randomization tests
\end{abstract}

O gênero Ogcocephalus Fischer, 1813 compreende 12 espécies, exclusivas do Novo Mundo, das quais duas são endêmicas em ilhas do Pacífico Oriental, enquanto as demais distribuem-se pelo Atlântico Ocidental (BRADBURY 1980).

Com base na revisão deste gênero realizada por BRADBURY (1980), três espécies ocorrem no litoral brasileiro: O. nasutus Cuvier \& Valenciennes, 1837, $O$. notatus Cuvier \& Valenciennes, 1837 e O. vespertilio (Linnaeus, 1758). Posteriormente, RAMOS \& VASCONCELLOS FILHO (1987) registraram também a ocorrência de $O$. declivirostris Bradbury, 1980 no litoral de Pernambuco. O peixe-morcego, Ogcocephalus vespertilio (L.), ocorre da foz do Rio Amazonas à foz do Rio da Prata, desde a costa até $144 \mathrm{~m}$ de profundidade (FIGUEIREDO \& MENEZES 1978). Apesar de sua ampla área de distribuição, pouco se conhece sobre a variação geográfica desta espécie. MENEZES (1964) realizou um estudo morfométrico de O. vespertilio, utilizando análise de regressão para comparar caracteres quantitativos de exemplares coletados em diferentes pontos do litoral brasileiro. LEMA \& PEREIRA (1975) compararam qualitativamente exemplares de $O$. vespertilio procedentes de Santa Catarina e Rio Grande do Sul, no limite meridional de distribuição da espécie.

1) Departamento de Biologia Geral, Universidade Santa Úrsula. Rua Fernando Ferrari 75 , 22231-040 Rio de Janeiro, Rio de Janeiro, Brasil.

2) Departamento de Ciências Biológicas, Universidade Estadual de Feira de Santana. Campus Universitário, BR 116 Km 3, 44031-460 Feira de Santana, Bahia, Brasil. 
Os métodos estatísticos convencionais de análise univariada ou bivariada principalmente a análise de variância e a análise de regressão - mostram-se inadequados à análise de padrões de variação geográfica em populações naturais, os quais são suficientemente complexos para que sua descrição e interpretação requeiram o uso das técnicas mais sofisticadas de análise estatística multivariada (GOULD \& JOHNSTON 1972; THORPE 1976, 1983; Willig et al. 1986; WILLIG \& OWEN 1987; REIS 1988), as quais permitem considerar os padrões de variação e covariação simultâneos de um conjunto de caracteres quantitativos. No Brasil, os estudos de variação geográfica em peixes, utilizando métodos de estatística multivariada, são bastante recentes e têm sido restritos a espécies de água doce (GARAVELLO et al. 1992; SHiBATTA \& GARAVELlo 1993). Os estudos sobre variação geográfica de peixes marinhos da costa brasileira, por outro lado, têm-se utilizado apenas de comparações através de técnicas convencionais de estatística descritiva (VAZZOLER 1971; Vargas 1976; Yamaguti 1979; Braga 1987; Paiva Filho \& Cergole 1988).

Pouco se conhece sobre os padrões de variabilidade em populações de peixes do Atlântico Ocidental, o que dificulta a compreensão dos fatores responsáveis pela diferenciação desta ictiofauna. Hipóteses biogeográficas (BALL 1975) poderiam ser derivadas de padrões de variação morfológica entre populações; contudo, até o presente nenhuma análise de variação geográfica em espécies de peixes do Atlântico Ocidental foi realizada com um enfoque em técnicas estatísticas adequadas à investigação destes padrões, como a realizada por MCEACHRAN et al. (1989) em raias pertencentes à família Rajidae no Atlântico Oriental.

Este trabalho tem por objetivo testar a hipótese da existência de variação geográfica em $O$. vespertilio no litoral brasileiro, utilizando métodos de análise estatística multivariada para avaliar os padrões de variação morfométrica entre populações selecionadas desta espécie.

\section{MATERIAL E MÉTODOS}

Foram analisados 91 exemplares de $O$. vespertilio provenientes dos litorais do Estado da Bahia (Baía de Todos os Santos, Ilha de Itaparica e Caravelas-Nova Viçosa), Estado do Espírito Santo (Guarapari) e Estado do Rio de Janeiro (Arraial do Cabo, Araruama-Cabo Frio, Baía de Guanabara, Ilhas Tijucas e Ilha Grande). (Fig. 1). Os exemplares examinados encontram-se depositados nas coleções do Laboratório de Citogenética de Peixes da Universidade Federal do Rio de Janeiro (UFRJ), Universidade do Estado do Rio de Janeiro (UERJ), Museu Nacional da Universidade Federal do Rio de Janeiro (MNRJ) e Universidade Estadual de Feira de Santana (UEFS).

Para cada exemplar, foram medidos 10 caracteres morfométricos (Fig. 2), tomados de acordo com MENEZES (1964), por meio de um paquímetro de aço com precisão de 0,05 mm: comprimento do rostro (ROS); diâmetro orbital (DO); comprimento da cavidade do ilício (CI); largura da cavidade do ilício (LI); espaço interorbital anterior (DIA); espaço interorbital posterior (DIP); distância do ânus à base da caudal ( $\mathrm{AC}$ ); distância do ânus à mandíbula $(\mathrm{AM})$; distância do ânus à base da anal (AA); comprimento-padrão (CP). 


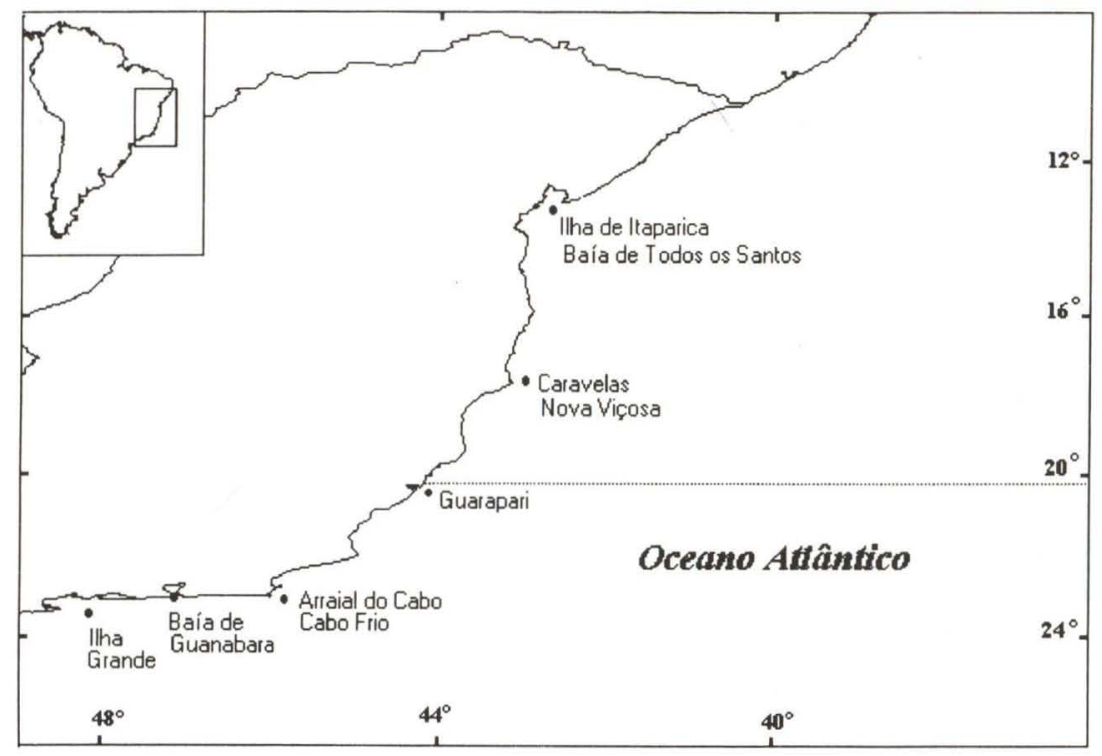

Fig. 1. Localidades amostradas no litoral brasileiro. A linha pontilhada indica o limite aproximado da isoterma de $23^{\circ} \mathrm{C}$.
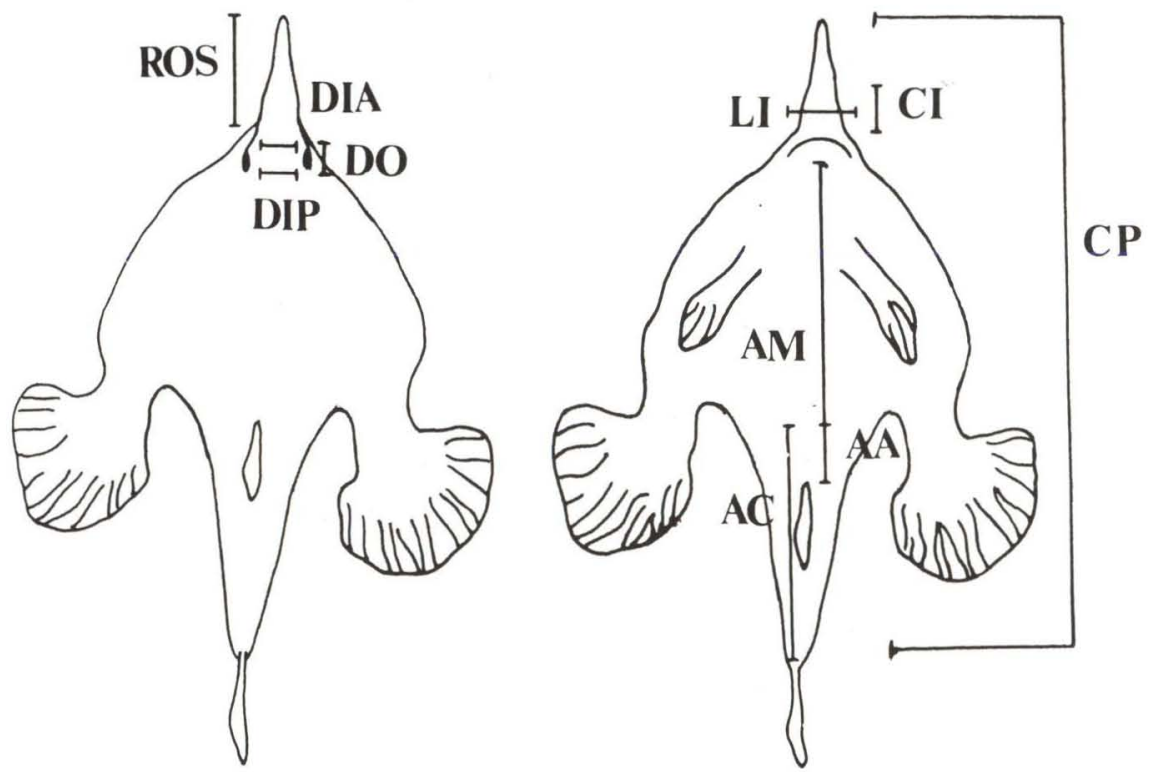

Fig. 2. Diagrama de O. vespertilio, indicando os caracteres morfometricos analisados. (ROS) Comprimento do rostro; (DO) diâmetro orbital; (Cl) comprimento da cavidade do ilício; (LI) largura da cavidade do ilício; (DIA) espaço interorbital anterior; (DIP) espaço interorbital posterior; (AC) distância do ânus à base da caudal; (AM) distância do ânus à mandíbula; (AA) distância do ânus à base da anal; (CP) comprimento-padrăo. 
Para a análise dos dados, a área amostrada foi dividida em duas regiões, localizadas, respectivamente, ao norte da isoterma de $23^{\circ} \mathrm{S}$, compreendendo as amostras da costa Nordeste (Bahia, com 18 exemplares), e ao sul desta isoterma, compreendendo as amostras da costa Sudeste (Espírito Santo e Rio de Janeiro, com 73 exemplares).

Esta divisão foi feita com base na proposição de PALACIO (1982) de que a isoterma de $23^{\circ} \mathrm{C}$ (linha de temperatura média constante durante todo o ano, situada aproximadamente na latitude de $21^{\circ} \mathrm{S}$ ) define o limite entre duas regiões biogeográficas distintas da costa brasileira, e na sugestão de LEMA \& PEREIRA (1975) de que a distribuição de $O$. vespertilio pode ser influenciada pela temperatura da água.

Os dados morfométricos obtidos das amostras de cada região foram submetidos ao tratamento estatístico básico, computando-se as médias (com seus respectivos desvios-padrões), e os valores máximos e mínimos, para cada um dos caracteres.

A análise de componentes principais (NEFF \& MARCUS 1980; BOOKSTEIN et al. 1985; MARCUS 1990; MORRISON 1990) foi utilizada para avaliar os padrões de discriminação entre as amostras. Esta análise foi efetuada sobre a matriz de covariâncias computada entre as variáveis morfométricas, a partir dos dados transformados em logaritmos decimais, a fim de normalizar sua distribuição e homogeneizar as variâncias (SOKAL \& ROHLF 1995). Desta matriz, foram extraídos os autovetores e seus autovalores associados, sendo os dados originais combinados aos autovetores e projetados sobre os três primeiros eixos ortogonais resultantes. Estes eixos, mutuamente perpendiculares e não correlacionados entre si, explicam sucessivamente a maior parte da variação presente nos dados originais, possibilitando (1) avaliar as relações entre os indivíduos, estabelecidas por sua proximidade no espaço delimitado pelo componentes principais e (2) reconhecer o valor discriminatório dos caracteres com respeito a essas relações (NEFF \& MARCUS 1980; BOOKSTEIN et al. 1985). A análise de componentes principais não exige que os grupos em estudo sejam definidos a priori e, portanto, permite verificar a presença de grupos distintos em um conjunto de dados (GOULD \& JOHNSTON 1972; HUMPHRIES et al. 1981). Gráficos vetoriais (MARCUS 1993) foram usados para representar as direções de variação máxima dos caracteres, no espaço dos dois primeiros componentes principais.

A análise de componentes principais, contudo, é uma técnica de ordenação exploratória e não permite testar a significância estatística dos grupos em estudo. No presente estudo, a hipótese da existência de diferenças estatisticamente significantes entre os valores amostrais médios para cada região, considerando todos os caracteres simultaneamente, foi testada contra a hipótese nula da inexistência de diferenças, através da análise de variância multivariada (Manova) e da estatística $\lambda$ de Wilks (NeFF \& MARCUS 1980; MARCUS 1990; MorRISON 1990). Um teste de randomização (MANLY 1991; CROWLEY 1992; SOKAL \& ROHLF 1995), com 5.000 permutações aleatórias dos exemplares entre os grupos em estudo, foi usado para calcular a probabilidade exata da estatística $\lambda$, ao nível de significância de $1 \%$ (MANLY 1991; CROWLEY 1992). Esta técnica é independente de suposições sobre a distribuição de probabilidades das variáveis mensuradas e, com a maior disponi- 
bilidade dos microcomputadores, tem-se demonstrado um procedimento bastante útil na validação de testes de significância estatística em estudos morfométricos (MANLy 1991; CROWLEY 1992; LYNCH 1996).

As análises estatísticas univariadas e multivariadas foram efetuadas em um microcomputador da linha IBM-PC, através do pacote estatístico SYSTAT, versão 5.03 (WiLKINSON 1990), exceto o cálculo da estatística $\lambda$ de Wilks com teste de randomização, que foi realizado com o programa Multivar (CAVALCANTI 1995), escrito em BASIC e disponível mediante solicitação.

\section{RESULTADOS}

As estatísticas descritivas básicas computadas para as amostras de cada região encontram-se na tabela I.

Tabela I. Estatísticas descritivas (média, desvio-padrão e amplitude) para os caracteres morfométricos (em milímetros) analisados. (ROS) Comprimento do rostro; (DO) diâmetro orbital; (CI) comprimento da cavidade do ilício; (LI) largura da cavidade do ilício; (DIA) espaço interorbital anterior; (DIP) espaço interorbital posterior; (AC) distância do ânus à base da caudal; (AM) distância do ânus à mandíbula; (AA) distância do ânus à base da anal; (CP) comprimento-padrão).

\begin{tabular}{lrrrrrrrrr}
\hline Caracteres & \multicolumn{3}{c}{ Costa Nordeste $(\mathrm{n}=18)$} & & \multicolumn{3}{c}{ Costa Sudeste $(\mathrm{n}=73)$} \\
\cline { 2 - 6 } \cline { 7 - 9 } & & Média \pm Desvio & Mínimo & Máximo & & Média \pm Desvio & Mínimo & Máximo \\
\hline ROS & $16,52 \pm$ & 6,00 & 8,50 & 30,95 & & $12,60 \pm$ & 3,78 & 5,10 & 26,20 \\
DO & $4,80 \pm$ & 1,46 & 1,75 & 8,15 & & $5,93 \pm$ & 1,08 & 1,70 & 8,80 \\
CI & $6,15 \pm$ & 2,40 & 2,80 & 11,30 & & $6,29 \pm 1,48$ & 2,15 & 12,15 \\
LI & $1,87 \pm$ & 1,19 & 0,35 & 4,80 & & $2,95 \pm 0,75$ & 0,35 & 4,20 \\
DIA & $8,19 \pm$ & 2,62 & 4,60 & 13,10 & & $8,13 \pm 1,80$ & 1,85 & 13,00 \\
DIP & $10,75 \pm$ & 3,28 & 5,15 & 16,75 & & $11,32 \pm 2,52$ & 3,50 & 18,30 \\
AC & $34,95 \pm 11,36$ & 19,50 & 52,50 & & $32,69 \pm 9,05$ & 11,70 & 59,10 \\
AM & $51,22 \pm 15,47$ & 32,30 & 82,65 & & $49,72 \pm 14,09$ & 22,35 & 87,80 \\
AA & $20,76 \pm 14,60$ & 9,20 & 72,80 & & $16,77 \pm 4,80$ & 5,90 & 29,50 \\
CP & $99,83 \pm 29,03$ & 59,65 & 157,40 & & $91,86 \pm 26,90$ & 39,75 & 175,00 \\
\hline
\end{tabular}

Os três primeiros eixos obtidos na análise de componentes principais explicaram cerca de $93,9 \%$ da variação total presente na matriz de covariâncias entre os caracteres. O primeiro componente principal explicou cerca de 70,3\% da variação total, enquanto o segundo deu conta de $20,9 \%$ e o terceiro cerca de $2,7 \%$ (Tab. II). Os coeficientes do primeiro autovetor apresentaram valores positivos para todas as variáveis morfométricas analisadas, podendo-se interpretar este autovetor como um fator de tamanho geral (BOOKSTEIN et. al. 1985). O segundo e o terceiro autovetores apresentaram coeficientes positivos e negativos, expressando informação sobre as diferenças de forma corporal entre as populações (Tab. II).

O gráfico da projeção dos indivíduos no espaço dos dois primeiros componentes principais mostra a presença de dois grupos diferenciados em relação à forma, compostos, respectivamente, pelas amostras do litoral da Bahia e do litoral do Rio 
de Janeiro, notando-se, porém, a sobreposição de alguns indivíduos desta última amostra, em relação aos da primeira (Fig. 3). A distribuição dos escores individuais no segundo e terceiro componentes principais, por outro lado, mostra que as amostras das duas regiões sobrepõem-se amplamente no espaço definido por estes componentes (Fig. 4) e, assim, não apresentam grande diferenciação quanto aos fatores relacionados apenas à forma.

Tabela II. Coeficientes dos três primeiros componentes principais (CP1, CP2, CP3) para os 10 caracteres morfométricos analisados, com seus respectivos autovalores e contribuiçöes percentuais.

\begin{tabular}{lrrr}
\hline \multicolumn{1}{c}{ Caracteres } & CP1 & CP2 & CP3 \\
\hline ROS & 0,112 & 0,064 & $-0,005$ \\
DO & 0,093 & $-0,042$ & $-0,017$ \\
CI & 0,102 & $-0,008$ & $-0,063$ \\
LI & 0,166 & $-0,156$ & 0,021 \\
DIA & 0,118 & 0,002 & 0,001 \\
DIP & 0,112 & 0,007 & $-0,005$ \\
AC & 0,113 & 0,042 & 0,015 \\
AM & 0,103 & 0,050 & 0,009 \\
AA & 0,129 & 0,051 & 0,016 \\
CP & 0,104 & 0,057 & 0,008 \\
\hline Autovalor & 0,136 & 0,041 & 0,005 \\
Porcentagem & 70,300 & 20,900 & 2,700 \\
\hline
\end{tabular}
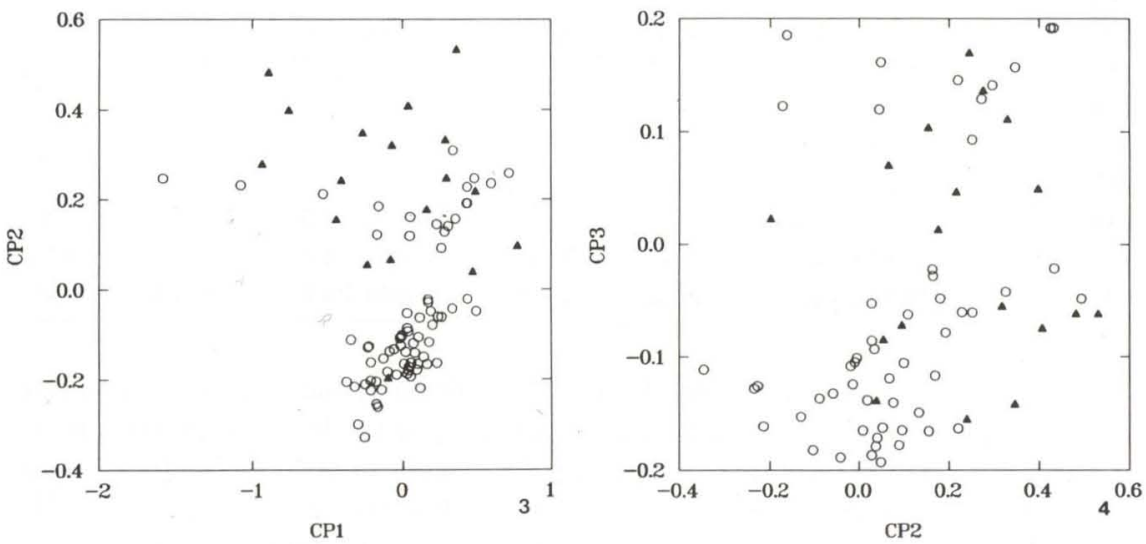

Figs 3-4. Projeção dos escores dos individuos de O. vespertilio. (3) No espaço dos dois primeiros componentes principais; (4) no espaço dos segundo e terceiro componentes principais. (A) Costa Nordeste, (O) costa Sudeste).

As variáveis que mais contribuíram para a diferenciação das populações na análise de componentes principais foram a largura da cavidade do ilício e a distância do ânus à origem da nadadeira anal, em relação aos dois primeiros componentes 
principais, enquanto que o comprimento e a largura da cavidade do ilício apresentaram as maiores contribuições para o terceiro componente principal (Tab. II). O segundo componente principal representa um contraste entre o comprimento e a largura da cavidade do ilício e o diâmetro orbital, que apresentaram coeficientes negativos, e os demais caracteres, que apresentaram coeficientes positivos neste componente (Tab. II, Fig. 5). O terceiro componente principal mostra um contraste entre o comprimento do rostro, o diâmetro orbital, o comprimento e a largura da cavidade do ilício e a distância do espaço interorbital posterior, com coeficientes negativos, e os demais caracteres, com coeficientes positivos (Tab. II).
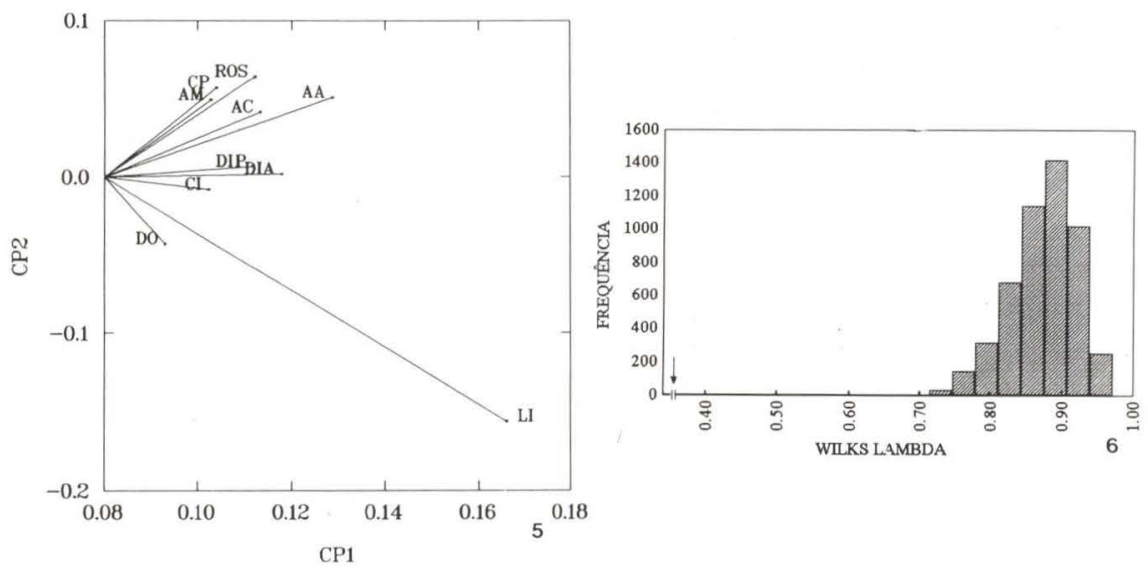

Figs 5-6. (5) Vetores de maior variação dos caracteres morfométricos analisados no espaço dos dois primeiros componentes principais; (6) histograma dos valores da estatística $\lambda$ de Wilks obtidos em 5.000 permutações aleatórias dos exemplares entre os grupos em estudo. A seta indica o valor de $\lambda$ obtido para os dados originais.

O valor do teste multivariado de Wilks obtido para os dados originais $(\lambda=$ 0,34083 ) foi menor do que todos os valores calculados para 5.000 permutações aleatórias dos exemplares entre os grupos estudados (Fig. 6). A diferença entre as regiões, considerando-se todos os caracteres morfométricos simultaneamente, foi altamente significativa $(\mathrm{P}=0,0002)$.

\section{DISCUSSÃO}

Os padrões de discriminação morfométrica entre as populações de $O$. vespertilio analisadas sugerem a existência de dois grupos de populações morfologicamente distintas, um deles compreendendo as populações da costa Nordeste (litoral da Bahia) e a outra compreendendo as populações da costa Sudeste (litorais do Espírito Santo e Rio de Janeiro). A diferenciação destes grupos está primariamente relacionada à largura da cavidade do ilício e, em menor grau, à distância do ânus à base da nadadeira anal, cujas dimensões tendem a ser maiores nos exemplares provenientes da costa Nordeste do que naqueles da costa Sudeste. 
A diferenciação morfológica entre as populações da costa Nordeste, assim como entre as populações do litoral do Rio de Janeiro, pode refletir a atuação de fatores ambientais. A região que se estende entre o Rio de Janeiro e o Espírito Santo marca o limite da isoterma de $23^{\circ} \mathrm{C}$ (PALACIO 1982), a qual pode atuar como uma barreira sobre muitas espécies marinhas, facilitando os processos de diversificação. Outros estudos de diferenciação geográfica de peixes marinhos realizados na costa sudeste-sul do Brasil (VAZZOLER 1971; VARGAS 1976; YAMAGUTI 1979; BRAGA 1987; Paiva Filho \& Cergole 1988), bem como na costa oeste da África (KOMPOWSKI 1975; MCEACHRAN et. al. 1989), também sugeriram a existência de populações distintas. Em todos estes estudos, as variações de temperatura da água, ocasionadas tanto por fenômenos de ressurgência como por influência de diferentes correntes marinhas, foram apontados como o principal fator responsável pelo isolamento geográfico e consequente redução do fluxo gênico entre as populações. A temperatura da água é um dos fatores ecológicos de maior importância para a distribuição da fauna marinha (EKMAN 1953; VANNUCCI 1964; BRIGGS 1974; PALACIO 1982), sendo também reconhecido como uma das principais causas de alterações morfológicas em populações de peixes (BARLOW 1961). As variações na temperatura da água, juntamente com variações na direção das correntes, parecem determinar o isolamento geográfico e a formação de novas espécies no ambiente marinho (BRIGGs 1974; PALUMBI 1994). Com relação a O. vespertilio, LEMA \& PEREIRA (1975) assinalam que a abundância da espécie no extremo sul do Brasil é maior na primavera e no verão, diminuindo no outono e inverno, e atribuem essas flutuações à variação sazonal das correntes marinhas predominantes na região.

Os resultados deste estudo indicam a existência de variação geográfica em $O$. vespertilio na costa do Brasil, mas análises posteriores, possivelmente utilizando métodos bioquímicos, são recomendadas para elucidar seu significado evolutivo nesta espécie.

AGRADECIMENTOS. Agradecemos a Mario Jorge Ignacio Brum (UFRJ), Ulisses Leite Gomes (UERJ) e Gustavo Wilson Nunan (MNRJ) pela permissão em utilizar o material depositado nas coleções ictiológicas sob seus cuidados; Acácio Ribeiro Tomás (Instituto de Pesca, Santos, SP), pela doação de exemplares de O. vespertilio; John M. Lynch (Arizona State University), que forneceu o estímulo inicial para o desenvolvimento do programa MULTIVAR; J. Becker (MNRJ) e um revisor anônimo pela leitura crítica do texto e valiosas sugestões, que em muito contribuíram para o aperfeiçoamento do manuscrito. Os autores permanecem, contudo, responsáveis por quaisquer erros ou omissões.

\section{REFERÊNCIAS BIBLIOGRÁFICAS}

BALL, I.R. 1975. Nature and formulation of biogeographical hypotheses. Syst. Zool. 24: 407-430.

BARLOW, G.W. 1961. Causes and significance of morphological variation in fishes.

Syst. Zool. 10: 105-117.

BoOKSTEIN, F.L.; B. ChernofF; R.L. Elder; J.M. HumPhries JR., G.R. SMITH \&

R.E. STRAUSS. 1985. Morphometrics in Evolutionary Biology. Special Pub-

lication 15. Philadelphia, The Academy of Natural Sciences, XVII $+277 \mathrm{p}$.

BRADBURY, M.G. 1980. A revision of the fish genus Ogcocephalus with descripti- 
ons of new species from the Western Atlantic Ocean (Ogcocephalidae: Lophiiformes). Proc. Calif. Acad. Sci. 42: 229-285.

BRAGA, F.M.S. 1987. Estudo da diversidade de Sardinella brasiliensis (Steindachner, 1879), na área entre Macaé $\left(22^{\circ} 23^{\prime} \mathrm{S}\right)$ e Ilha de Santa Catarina $\left(27^{\circ} 35^{\prime} \mathrm{S}\right)$.

1. Crescimento de dimensões corporais. Revta Bras. Zool. 4: 235-250.

BRIGGS, J.C. 1974. Marine Zoogeography. New York, McGraw-Hill, XI+475p. CAVALCANTI, M.J. 1995. Multivar - Comparison of multivariate samples with randomization tests. MS-DOS software, Departamento de Biologia Geral, Universidade Santa Úrsula, Rio de Janeiro.

CROWLEY, P.H. 1992. Resampling methods for computation-intensive data analysis in ecology and evolution. Ann. Rev. Ecol. Syst. 23: 405-447.

EKMAN, S. 1953. Zoogeography of the Sea. London, Sidgwick and Jackson, $\mathrm{XIV}+417 \mathrm{p}$.

Figueiredo, J.L. \& N.A. Menezes. 1978. Manual de Peixes Marinhos do

Sudeste do Brasil. II. Teleostei (1). São Paulo, Museu de Zoologia da Universidade de São Paulo, 110p.

Garavello, J.C.; S.F. DOS ReIS \& R.E. Strauss. 1992. Geographic variation in Leporinus friderici (Bloch) (Pisces: Ostariophysi: Anostomidae) from the Paraná-Paraguay and Amazon River basins. Zool. Scr. 21: 197-200.

Gould, S.J. \& R.F. Johnston. 1972. Geographic variation. Ann. Rev. Ecol. Syst. 3: $457-498$.

Humphries, J.M.; F.L. BoOKSTEIn; B. ChernofF; G.R. SMith; R.L. Elder \& S.G. Poss. 1981. Multivariate discrimination by shape in relation to size. Syst. Zool. 30: 291-308.

KoMPOWSKI, A. 1975. The intraspecific geographical variability of horse mackerel Trachurus trachurus (L.) in the West African shelf waters. Acta Ichthyol. Piscat. 5: 13-29.

Lema, T. \& J. PEREIRA JR. 1975. Sobre Ogcocephalus vespertilio (L., 1758) ocorrente no extremo sul do Brasil (Teleostei, Lophiiformes, Ogcocephalidae). Iheringia, Sér. Zool., 48: 75-86.

LYNCH, J.M. 1996. Sexual dimorphism in cranial size and shape among red foxes Vulpes vulpes from north-east Ireland. Proc. Royal Irish Acad. 96B: 21-26.

MANLY, B.F.J. 1991. Randomization and Monte Carlo Methods in Biology. London, Chapman and Hall, XIII+281p.

MARCUS, L.F. 1990. Traditional morphometrics, p.77-122. In: F.J. RoHLF \& F.L. BOOKSTEIN (Eds). Proceedings of the Michigan Morphometrics Workshop. Ann Arbor, The University of Michigan Museum of Zoology, Special Publication No. 2, VIII+380p.

1993. Some aspects of multivariate statistics for morphometrics, p.95-130. In: L.F. MARCUS; E. Bello \& A. García-VAldeCASAS (Eds). Contributions to Morphometrics. Madrid, Museu Nacional de Ciencias Naturales, 264p.

MCEACHRAN, J.D., B. SÉRET \& T. MIYAKE. 1989. Morphological variation within Raja miraletus and status of $R$. ocellifera (Chondrichthyes, Rajoidei). Copeia 1989: 629-641.

MenEZES, N. 1964. Sobre Ogcocephalidae das costas do Brasil (Pisces, Ogcocephalidae). Papéis Avulsos Zool., São Paulo, 16: 153-171.

MORRISON, D.F. 1990. Multivariate Statistical Methods. New York, McGraw-

Revta bras. Zool. 15 (1): 125 - 134, 1998 
Hill, XVII+495p.

NefF, N.A. \& L.F. Marcus. 1980. A Survey of Multivariate Methods for

Systematics. New York, Privately published, X+243p.

PALACIO, F.J. 1982. Revisión zoogeográfica marina del sur del Brazil. Bolm. Inst.

Oceanogr., São Paulo, 31: 69-92.

PALUMBI, S.R. 1994. Genetic divergence, reproductive isolation, and marine speciation. Ann. Rev. Ecol. Syst. 25: 547-572.

Paiva Filho, A.M. \& M.C. Cergole. 1988. Diferenciação geográfica de Nebris micropis (Cuvier, 1830) na costa sudeste do Brasil. Bol. Inst. Oceanogr., São Paulo, 36: 37-45.

RAmos, R.T.C. \& A.L. VASCONCElos FilHo. 1987. Novas ocorrências de peixes demersais para a costa nordeste do Brasil. Trab. Oceanogr. Univ. Fed. Pernambuco 20: 197-202.

REIS, S.F. 1988. Morfometria e estatística multivariada em biologia evolutiva. Revta bras. Zool. 5: 571-580.

ShibattA, O.A. \& J.C. Garavello. 1993. Estudo da variação geográfica em Salminus hilarii Valenciennes, 1849, das Bacias do Alto Paraná e São Francisco, através da análise morfométrica multivariada em componentes principais. Naturalia 18: 109-116.

SoKal, R.R. \& F.J. RoHlF. 1995. Biometry: The Principles and Practice of Statistics in Biological Research. San Francisco, W.H. Freeman, $3^{\text {rd }}$ ed., $\mathrm{XIX}+887 \mathrm{p}$.

THORPE, R.S. 1976. Biometric analysis of geographic variation and racial affinities.

Biol. Rev. 51: 407-452.

1983. A review of the numerical methods for recognising and analysing racial differentiation, p.404-423. In: J. FELSENSTEIN (Ed.). Numerical Taxonomy. Nato Advanced Study Institute Series G (Ecological Sciences), No. 1. Berlin, Springer-Verlag.

VAnNuCCI, M. 1964. Zoogeografia marinha do Brasil. Bolm. Inst. Biol. Mar. 7: 113-121.

VARGAS, C.P. 1976. Geographic differentiation of Paralonchurus brasiliensis (Steindachner, 1875) between the latitudes $23^{\circ} 30^{\prime} \mathrm{S}$ (Ubatuba, SP) and $32^{\circ} \mathrm{S}$ (Barra do Rio Grande, RS), Brazil. Revue Trav. Inst. Pêch. Marit. 40: 775-779. VAZZOLER, A.E.A.M. 1971. Diversificação fisiológica e morfológica de Micropogon furnieri (Desmarest, 1822) ao sul de Cabo Frio, Brasil. Bol. Inst. Oceanogr., S. Paulo, 20: 1-70.

WILkINSON, L. 1990. SYSTAT: The System for Statistics. Evanston, SYSTAT Inc., XVIII+677p.

WILLIG, M.R. \& R.D. OWEN. 1987. Univariate analyses of morphometric variation do not emulate the results of multivariate analyses. Syst. Zool. 36: 398-400.

Willig, M.R.; R.D. OWEN \& R.L. COLBERT. 1986. Assessment of morphometric variation in natural populations: the inadequacy of the univariate approach. Syst. Zool. 35: 195-203.

YAMAGUTI, N. 1979. Diferenciação geográfica de Macrodon ancylodon (Bloch \& Schneider, 1801) na costa brasileira, entre as latitudes $18^{\circ} 36^{\prime} \mathrm{S}$ e $32^{\circ} 10^{\prime} \mathrm{S}$. Etapa I. Bol. Inst. Oceanogr., S. Paulo, 28: 53-118.

Recebido em 28.II.1997; aceito em 13.IV.1998. 